\title{
Historia de la mortalidad infantil en la Casa de Huérfanos de Santiago (1898-1923)*
}

\author{
History of infant mortality in the House \\ of Orphans of Santiago (1898-1923)
}

\author{
Pablo Chávez Zúñiga \\ ORCID iD: http://orcid.org/0000-0002-1726-7954 \\ Universidad de Chile \\ José Julián Soto Lara \\ ORCID iD: http://orcid.org/0000-0003-2263-1674 \\ Universidad Bernardo O’Higgins-Chile
}

\begin{abstract}
El estudio analiza las características de los niños ingresados en la Casa de Huérfanos entre 1898 y 1923, contextualizándolas históricamente, explicitando las razones del abandono según los adultos y delimitando los significados de la niñez. También aborda la discusión médica sobre la mortalidad infantil en la Casa de Huérfanos, la que permitió, en parte, el surgimiento de la pediatría chilena. Además, desarrolla las experiencias de los párvulos durante la admisión al recinto, condicionadas por el desarraigo y la pobreza. Las conclusiones tratan el proceso de medicalización infantil a través de la institución.
\end{abstract}

Palabras Clave: Niño; Abandono; Casa de Huérfanos; Mortalidad infantil; Madre.

The study analyzes the characteristics of those children that were admitted to the orphanage «Casa de Huérfanos» between 1898 and 1923, contextualizing them historically, explaining the reasons for abandonment given by the adults and demarcating the meanings of childhood. It also tackles the medical discussion on infant mortality in the "Casa de Huérfanos» which allowed, in part, the emergence of Chilean pediatrics. In addition, the experiences of kindergartens during the admission, conditioned by uprooting and poverty. The conclusions deal with the process of medicalization via the institution.

Keywords: Children; Abandonment; House of Orphans; Infant Mortality; Mother.

Copyright: (C) 2019 CSIC. Este es un artículo de acceso abierto distribuido bajo los términos de la licencia de uso y distribución Creative Commons Reconocimiento 4.0 Internacional (CC BY 4.0).

* Este artículo es un avance de investigación de la tesis doctoral «Esa diaria gotera de la muerte: mortalidad infantil en Santiago (1860-1914)», cuyo autor es Pablo Chávez Zúñiga, perteneciente al programa Doctorado en Historia en la Universidad de Chile. Agradecemos el financiamiento otorgado por la Comisión Nacional de Investigación Científica y Tecnología (CONICYT), a través de su programa formación de capital humano avanzado: Beca de Doctorado Nacional (2015) y Beca de Doctorado en el Extranjero «Becas Chile» (2017). Agradecemos los comentarios de los evaluadores. 


\section{Introducción}

El año 2016, la prensa chilena reveló la crisis humanitaria que atravesaba el Servicio Nacional de Menores (SENAME). La situación demostró la precariedad vivida por un segmento de la infancia chilena contemporánea. Entre 2004 y 2016, fallecieron 1.313 niños que estaban a cargo de la institución. La mayoría de ellos por discapacidades, descuidos y enfermedades. Esto generó un escándalo, donde todos los sectores políticos se responsabilizaron mutuamente, sin generar soluciones. Las raíces históricas del problema se encuentran en la creación del SENAME, en 1979, a partir de la reestructuración de varios servicios, entre ellos: la Fundación del Niño Chileno, el Consejo Nacional de Menores y la Casa Nacional del Niño.

En 1929, la Junta de Beneficencia Pública reemplazó el nombre de la Casa de Huérfanos por el de Casa del Niño, eliminando los prejuicios hacia los asilados e impidiendo la formación de una casta asociada a la orfandad. Tras esta perspectiva estuvo latente una concepción social que promovió la igualdad de los ciudadanos y de sus posibilidades para desarrollarse. Mediante el «Decreto Supremo n. ${ }^{\circ} 1340$, de 16 de julio de 1929, la antigua Casa de Huérfanos de Santiago pasó a llamarse Casa Nacional del Niño y toda Casa de Huérfanos de provincias, recibió el nombre de Casa del Niño». ${ }^{1}$ De esa manera, las instituciones estatales encargadas de la protección infantil en la actualidad, a pesar de las modificaciones nominales, tienen una conexión con aquella.

En este artículo analizamos el proceso de configuración de la Casa de Huérfanos de Santiago, relacionado con el contexto de mortalidad y abandono infantil. En ambas circunstancias destacaron la violencia y la ignorancia de los progenitores, quienes vieron en la institución una alternativa para la crianza de sus hijos. ${ }^{2}$ El dinamismo producido por la función social del organismo repercutió en el surgimiento de actores (familia, médicos, trabajadores de la beneficencia) que problematizaron la niñez. Durante el período estudiado, la medicina comenzó a ocuparse por los altos índices de mortalidad infantil. En particular, se interesó por las condiciones alimentarias e

1 Calvo Mackenna, 1933, 9.

2 En 1927, el doctor Luis Calvo Mackenna, para reformar la institución, reglamentó que todo niño abandonado sería aceptado bajo la expresa condición que estaría albergado en calidad de abandonado, sin derecho a ser reclamado. Calvo Mackenna, 1933, 7. Este cambio en el rol del orfanato respondió a su capacidad de rendimiento. El crecimiento demográfico de Santiago aumentó la cantidad de niños atendidos, muchos de los cuales eran ingresados transitoriamente. Por ello, era necesario limitar el acceso a determinadas situaciones de los infantes. 
higiénicas de los huérfanos, produciendo avances médicos que se aplicaron en el establecimiento.

Las investigaciones principales sobre la Casa de Huérfanos, el abandono y la infancia analizan la demografía histórica de los expósitos. ${ }^{3}$ Asimismo, sugieren que, probablemente, la internación infantil solo posponía la muerte de los niños. ${ }^{4}$ Igualmente, han enriquecido la mirada cultural de la crianza de los niños abandonados, ${ }^{5}$ profundizando en los significados de la familia, de los valores tradicionales y de la constitución de las instituciones receptoras. Otros historiadores han reflexionado sobre el uso popular dado a los establecimientos; las denuncias médicas contra la alta mortalidad de los internos, lo que permitió legislar al respecto, y el plano moral de la ilegitimidad para explicar la muerte de los neonatos. ${ }^{6}$

La historiografía latinoamericana ha estudiado los vínculos entre la mortalidad infantil y el abandono. En diferentes países, las migraciones campo-ciudad de mediados del siglo XIX produjeron una preocupación hacia los niños, quienes serían los futuros ciudadanos. En efecto, la acción del Estado se tradujo en distanciarlos de la criminalidad y el maximalismo. ${ }^{7}$

En Chile, los estudios han analizado la circulación de párvulos entre las instituciones y los parientes, así como las características de quienes permanecieron en estas. ${ }^{8}$ En el ámbito cultural, la historiografía ha subrayado las modificaciones de la afectividad materna hacia los hijos, traducidas en un repudio al infanticidio y al aborto, fortaleciéndose la alternativa de entregar infantes a la Casa de Huérfanos. En esa línea, la niñez abandonada ha sido tratada en larga duración (siglos XVIII y XIX), mostrando estadísticamente la procedencia de los expósitos (Santiago y provincias). ${ }^{9}$

Cronológicamente, el período analizado obedece a dos razones. En 1898, el doctor Roberto del Río cumplió una década a cargo de la cátedra

3 Los conceptos expósito y huérfano se deben matizar. El primero, proviene del latín expositus que significa expuesto, «poner fuera». Esta categoría comprendía a los recién nacidos que eran abandonados. El segundo, abarcaba a los párvulos que habían quedado desamparados por diversas circunstancias: fallecimiento de los padres, búsqueda de empleo o dificultades económicas, entre otras.

4 Martín-Espinosa, Villena y Cobo-Cuenca, 2016.

5 Ver: Dalla-Corte y Piacenza, 2006; Dalla-Corte, 2013; Osta Vásquez, 2016, 155-189; Franco, 2016, 437-469.

6 Boswell, 1988; Volker, 1991; Rodríguez, 1998, 19-52; Revuelta, 2011; Revuelta, 2012, 645672; Revuelta, 2013, 44-67; Revuelta, 2015, 107-130.

7 Para el caso latinoamericano, véase: González, 2000; Guy, 2000; Cowen, 2004; Rodríguez y Manarelli, 2007; Blum, 2010; Álvarez y Reynoso, 2011; Sánchez, 2014.

8 Milanich, 2001, 79-100; Milanich, 2004, 311-340; Milanich, 2009; Milanich, 2011, $29-62$.

9 Salinas y Delgado, 1990, 44-54; Salinas, 1991, 315-329; Delgado, 2001, 101-126; Palma, 2009, 127-137. 
de enfermedades de niños. ${ }^{10}$ En la práctica, la formación académica de los especialistas en medicina infantil se traduciría en la aplicación de conocimientos pediátricos en el recinto, lo que años más tarde mejoraría la sanidad de los infantes. Además, en 1901, se instaló transitoriamente el primer hospital de niños de Chile.

En el otro extremo, durante la década de 1920, la elite tomó conciencia sobre la mortalidad infantil como problema nacional, produciendo cambios en las políticas socio-infantiles. En ese contexto, las leyes de la infancia desvalida (1912), salas cunas (1917) e instrucción primaria obligatoria (1920), evidenciaron la necesidad de reformar las circunstancias de trabajo y el abandono infantil. A ello, se agregaron algunas mejoras en el hospital de niños impulsadas por Luis Calvo Mackenna que, años más tarde, llevaron a la implementación de instalaciones especializadas para el tratamiento de afecciones en la Casa de Huérfanos. De ese modo, el marco temporal de esta investigación abarca un período de diagnósticos médicos e implementación de medidas contra la mortalidad infantil.

La relación entre mortalidad infantil y la Casa de Huérfanos evidencia la numerosa tasa de ingreso de niños en esa institución, la práctica del abandono infantil y la conformación de un centro productor de saberes médicos para niños, que tuvo un radio de aplicación nacional.

En esta investigación proponemos cuatro objetivos: a) desarrollar la labor realizada por los médicos en la Casa de Huérfanos, atendiendo a sus debates en torno a la mortalidad infantil en la institución; b) Analizar los factores de los decesos de los recién nacidos, las condiciones y el período en que ingresaron al establecimiento; c) estudiar los vínculos entre el recinto, la cultura y la sociedad a partir de las notas elaboradas por las religiosas, quienes durante el ingreso del niño identificaron las diferentes circunstancias que provocaron su internación, y d) interrogar las relaciones entre el ejercicio práctico de la pediatría, el discurso médico de la mortalidad infantil y la constitución de la familia en Santiago. Este acercamiento aborda la medicalización que contribuyó a disminuir, drásticamente, los índices de mortandad.

10 El doctor Roberto del Río entró, en 1876, a la Facultad de Medicina de la Universidad de Chile, recibiendo el título de médico cirujano en 1883. Desde ahí se ocupó del estudio de las enfermedades infantiles. En 1907 fue nombrado Director del primer Hospital de Niños, cargo que desempeñó hasta su muerte, una década más tarde. En ese momento, la Junta de Beneficencia otorgó el nombre del especialista al recinto. Actualmente, el Hospital Roberto del Río es la institución de mayor prestigio y tecnología en el tratamiento pediátrico en Chile. 
Esos objetivos originaron las siguientes interrogantes: ¿Cuáles fueron las explicaciones sobre la mortalidad infantil en la Casa de Huérfanos por parte de los diversos actores sociales? ¿De qué manera las características de la institución ocasionaron anualmente las elevadas cifras de fallecimientos? ¿Cuál era el perfil de los internados? ¿Cómo impactó la urbanización en el aumento o disminución de la mortalidad infantil?

Sugerimos como hipótesis que la mortalidad infantil en la Casa de Huérfanos se enmarcó dentro de cambios institucionales modernizantes profundos (progresos de la medicina infantil) y culturales (narrativas nuevas del abandono), manifestados en la implementación de tratamientos médicos e higiénicos, con lo cual surgieron nuevas interrogantes a investigar con otros significados hacia la infancia.

\section{Método y fuentes}

La riqueza de los documentos elaborados por las Hermanas de la Providencia — denominados «Libros de ingresos de niños a la Casa de Huérfanos de Santiago»— permite reconstruir narrativas sobre el abandono, la mortalidad y la infancia. Sus páginas están divididas en tres columnas: en la primera aparece el nombre y una numeración correlativa a cada ingresado; en la segunda, el día de ingreso, lugar de procedencia, nombre de la persona que lo internó, nombre de los padres, legitimidad o no, edad de ingreso, descripción del bautizo, estado epidemiológico y algunos apuntes biográficos; en la tercera, el destino del ingresado.

Tras la transcripción, se construyó una base de datos con todos los infantes registrados entre julio de 1898 y agosto de $1923 .{ }^{11}$ La muestra se compone de 15.315 inscripciones; 449 incorporaron descripciones sucintas de los protagonistas o anotaciones institucionales; 579 constaron muertes maternas y 269 evidenciaron la existencia de hermanos. ${ }^{12}$ El detalle de la

11 A excepción de los registrados entre el 19 de octubre de 1902 y el 22 de junio de 1906, debido a la inexistencia de esos documentos en el Museo Nacional de la Medicina de la Universidad de Chile Doctor Enrique Laval.

12 El Código Penal graduaba las penas del delito de abandono según los actores, los hechos y las circunstancias que lo provocaban. La Ley no castigaba a los progenitores que dejaban niños en las Casas de Huérfanos, instituciones que protegían su vida e incrementaban su supervivencia. Siendo inexcusable el abandono de un menor que pusiera en riesgo su vida o salud, el artículo 346 del código estableció un presidio de hasta 540 días al que abandonare en un lugar no solitario a un niño menor 
información en los libros de registros ha permitido la formación de categorías presentadas en esta investigación, entre ellas, el número de ingresos por año, la relación entre la edad de entrada y el fallecimiento, y la distribución mensual de las muertes.

Los principales datos recogidos por las religiosas en el momento de elaborar los registros de ingresos de niños al establecimiento se expresan en el cuadro n. ${ }^{\circ}$ 1. Entre las características destacamos la aparición del nombre del padre o la madre. En este caso, la alta mención de mujeres sobre hombres como responsables del traslado de los párvulos hacia la Casa permite interpretar que esta fue una acción principalmente femenina. Pese a las constantes denominaciones en este aspecto, los libros de ingresos son limitados porque solo individualizaron a una persona, sin agregar otras informaciones como la dirección, el trabajo o los estudios, las que permitirían seguir las biografías de los niños. En ese marco, las anotaciones que efectuaron las religiosas adquieren importancia, ya que son los únicos antecedentes que permiten interpretar el abandono y las condiciones de los párvulos durante la admisión al recinto.

\section{CuAdro n. ${ }^{0} 1$}

LIBROS DE INGRESOS. CASA DE HUÉRFANOS DE SANTIAGO, 1898-1923

\begin{tabular}{|l|r|r|}
\hline \multicolumn{1}{|c|}{ Características } & Mención & Omisión \\
\hline Nombre padre & 3.818 & 11.468 \\
\hline Nombre madre & 14.366 & 920 \\
\hline Edad ingreso párvulo & 13.430 & 1.856 \\
\hline Agrega anotaciones institucionales & 449 & \\
\hline
\end{tabular}

Fuente: Libros de ingresos de niños a la Casa de Huérfanos de Santiago.

\footnotetext{
de siete años; en el caso que este acto lo ejecutaran los padres legítimos o ilegítimos o alguna persona que tuviera al niño bajo su cuidado, el artículo 347 otorgaba una pena de hasta cinco años, teniendo como agravante que el hecho se produjera a menos de cinco kilómetros de un pueblo o lugar donde hubiera una Casa de Expósitos. En la situación que se produjeran lesiones graves o la muerte del infante abandonado, el artículo 348 castigaba hasta con cinco años al delincuente. La paternidad era un agravante con penas que podían llegar hasta los diez años.
} 
La ficha de ingreso tipificó algunas discapacidades de los párvulos con las siguientes expresiones: sordo mudo, ${ }^{13}$ mudo, ${ }^{14}$ ciego,${ }^{15}$ defectuoso,${ }^{16}$ con pies defectuosos, ${ }^{17}$ pies torcidos. ${ }^{18}$ En estos registros los médicos, que solo hacían visitas semanales a los niños, no los diagnosticaban ni detectaban científicamente enfermedades, aumentando así la probabilidad de fallecimiento. Las fichas también manifestaban interés por el bautismo, es decir, si venía con ese sacramento. De lo contrario, la institución se lo proveía para salvarlo del pecado original. En este ámbito primaba la visión moral del Estado y la Iglesia. ${ }^{19}$

Los lugares principales donde se abandonaron niños fueron las acequias y calles, el cementerio y la Casa de Huérfanos. ${ }^{20}$ Por ejemplo, en 1919, el guardián Manuel Moreno trasladó a la sección lactantes de la Casa a los hermanos Elva y Gilberto, de cuatro y dos años, de padres desconocidos, hallados juntos en el barrio Matadero. Igualmente, el policía Adrián Ramírez aseguró que una pequeña hallada en la calle y enviada a la primera Comisaría, posteriormente, fue reclamada por sus progenitores. ${ }^{21}$ Estas situaciones muestran los circuitos del abandono, los lugares y las posibilidades de reclamar el retorno del párvulo a su familia.

También fueron anotados los objetos portados por los infantes al ingresar a la Casa. Entre ellos resaltan las medallas con motivos de San Benito, de San Antonio, del Sagrado Corazón, de la Santísima Virgen con el

13 Museo Nacional de la Medicina de la Universidad de Chile Doctor Enrique Laval, libros de ingresos de niños a la Casa de Huérfanos de Santiago, ingreso n. ${ }^{\circ} 1.227$ (02/12/1901).

14 Ingreso n. ${ }^{\circ} 9.409(13 / 06 / 1913)$.

15 Ingreso n. ${ }^{\circ} 14.604(21 / 08 / 1920)$

16 Ingreso n. ${ }^{\circ} 9.661(14 / 10 / 1913)$.

17 Ingreso n. ${ }^{\circ} 13.421(14 / 03 / 1919)$, n. $^{\circ} 15.785(10 / 03 / 1922)$.

18 Ingreso n. ${ }^{\circ} 14.941(16 / 12 / 1920)$.

19 Para la época, en España, la construcción del discurso médico explicaba la mortalidad de los infantes por las condiciones de los niños al ingresar a la inclusa y por tener un carácter ilegítimo, por ende, eran significados como hijos del vicio, la inmoralidad y los excesos de sus padres. Véase, Revuelta, 2015, 109. En el caso de España, la RAE define una inclusa como una casa donde se recoge y cría a los niños expósitos.

20 Las fichas de entrada, algunas veces, colocaron la observación del lugar donde fue encontrado abandonado un niño. Los que abordaron este ámbito son los siguientes: n. $^{\circ} 13.608$ (18/08/1898), n. ${ }^{o} 1.425$ (31/03/1902), n. ${ }^{o} 12.209$ (16/07/1917), n. ${ }^{\circ} 345$ (18/08/1900), n. ${ }^{\circ} 1.106(07 / 10 / 1901)$, n. $^{\circ} 9.876$ (27/01/1914), n. ${ }^{\circ} 10.108(26 / 06 / 1914)$, n. $^{\circ} 15.568$ (04/11/1921), n. ${ }^{\circ} 6.072$ (12/08/1908), n. ${ }^{\circ} 10.207$ (18/08/1914), n. ${ }^{\circ} 13.419$ (12/03/1919), n. ${ }^{\circ} 10.429$ (17/11/1914), n. ${ }^{\circ} 13.549$ (31/05/1919), n. ${ }^{\circ} 12.989$ (28/08/1918), n. ${ }^{\circ} 11.797$ (28/11/1916), n. ${ }^{\circ} 7.243$ (13/06/1910), n. ${ }^{\circ} 7.796(07 / 03 / 1911)$, n. $^{\circ} 8.054$ (05/08/1911), n. ${ }^{\circ} 11.104(20 / 11 / 1915)$, n. $^{\circ} 10.811$ (01/07/1915), n. ${ }^{\circ} 4.494(05 / 07 / 1906)$, n. $^{\circ} 11.889$ (13/01/1917).

21 Ingreso n. ${ }^{\circ} 10.230(29 / 08 / 1914)$. 
niño Jesús, de la Virgen del Carmen y de la Virgen del Perpetuo Socorro. ${ }^{22}$ En los casos revisados, probablemente, los padres colocaron estos objetos por el significado religioso. De igual modo, era destacado el ropaje: ropa fina, acompañado de un paquete con prendas, usadas o nuevas, envuelto en un pañuelo usado o en papel de diario. ${ }^{23}$ Asimismo hubo menores abandonados desnudos. ${ }^{24}$ En ocasiones, el recién nacido portaba un papel que indicaba la edad y una sugerencia de nombre. ${ }^{25}$ Edmundo, ingresado con poco más de un mes de vida y fallecido a los tres, portaba una carta «para entregársela cuando tenga 18 años». ${ }^{26} \mathrm{~A}$ partir de los datos consignados en las identificaciones se sabe que las religiosas colocaron nombres a los entrados, además las cifras de recuperación eran bajas, lo que supone que la reclamación no estaba entre las principales razones.

El número de internados en la Casa entre 1898-1923 se aprecia en el cuadro . $^{\circ}$ 2. En este caso se propuso la organización de la información en quinquenios porque genera muestras consolidadas con mayor cantidad de datos, a diferencia de un conjunto anual compuesto por cifras reducidas. El crecimiento sostenido fue condicionado por el aumento poblacional natural y por la migración campo-ciudad. ${ }^{27}$ Esos datos, cotejados con los de natalidad de la provincia de Santiago, ${ }^{28}$ permiten conocer el porcentaje de niños ingresados en la institución. A partir de la relación entre las entradas y las

22 Las anotaciones de los objetos materiales que acompañaban a los niños durante su admisión en la Casa se encuentran anotadas en los siguientes ingresos: $n{ }^{\circ} 13.574$ (09/08/1898), $n{ }^{\circ}$ 5.845 (12/04/1908), n. ${ }^{\circ} 8.501$ (16/02/1912), n. ${ }^{o} 15.070$ (19/02/1921), n. ${ }^{\circ} 9.419$ (18/06/1913), n. ${ }^{\circ} 8.496$ (14/02/1912), n. ${ }^{\circ} 9.820(24 / 12 / 1913)$, n. $^{\circ} 11.976(28 / 02 / 1917)$, n. $^{\circ} 9.652(11 / 10 / 1913)$, n. $^{\circ} 13.752$ $(02 / 11 / 1898)$.

23 Algunas inscripciones que consideraron la vestimenta de los menores cuando entraron al establecimiento: n. ${ }^{\circ} 14083$ (30/05/1899), n. 388 (16/09/1900), n. ${ }^{\circ} 1.642$ (04/08/1902), n. ${ }^{\circ} 8.958$ (11/10/1912), n. ${ }^{\circ} 9.024$ (02/11/1912), n. ${ }^{\circ} 9.232(20 / 02 / 1913)$, n. $^{\circ} 9.311(20 / 04 / 1913)$, n. $^{\circ} 9.673$ (19/10/1913), n. ${ }^{\circ} 9.684$ (22/10/1913), n. ${ }^{\circ} 9.665$ (15/10/1913), n. ${ }^{\circ} 12.958$ (14/08/1918).

24 Ingreso n. ${ }^{\circ} 10.838(13 / 07 / 1915)$.

25 Ingreso n. ${ }^{\circ} 13.842$ (17/12/1898). El Ferrocarril construía una imagen que se proyectaba desde la elite hacia el pueblo. En ella un párvulo encontrado con una tarjeta con «una mui buena letra; lo que significa que ya no es la clase infeliz i miserable del pueblo quien arroja sus hijos a la calle, las más veces compelida por su miseria misma». «Un párvulo», El Ferrocarril, Santiago, 28 de marzo de $1864,3$.

26 Ingreso n. ${ }^{\circ} 16.674(20 / 04 / 1923)$

27 Una de las consecuencias del proceso de revolución industrial fue el crecimiento demográfico de las capitales en pocas décadas. La provincia de Santiago aumentó su población, según los censos de 1885, 1895, 1907 y 1920, en: $326.671,414.494,515.789$ y 685.358 personas, respectivamente.

28 Total de nacidos en la Providencia de Santiago por año y porcentaje de menores abandonados en la Casa de Huérfanos: 18.602 (1909), 3,4 \%; 23.632 (1916), 2,8 \%; 25.278 (1917), 2,8 \%; 24.689 (1918), 2,9\%; 24.081 (1919), 3,3\%; 24.628 (1920), 3,5\%; 26.489 (1921), 2,6\%; 25.942 (1922), 2,9\%; 27.340 (1923), $2,1 \%$. 
defunciones, el cálculo de porcentaje de mortalidad bruta descendió desde $75.4 \%$ en el período inicial hasta el $59.7 \%$ en la parte final de la muestra. Igualmente, al considerar los ingresos de párvulos menores de un año y el número de fallecimientos, la mortalidad infantil disminuyó desde $80.8 \%$ al $76.4 \%$. Además, al examinar la composición etaria de los ingresados, se aprecia que el porcentaje de admisión de los menores de un año respecto al total, en el período 1898-1902, equivale al $91.6 \%$, la que va disminuyendo hasta llegar al $74.3 \%$, lo que relacionado con el número de acogidos menores de un mes permite suponer que el porcentaje de entrados varió desde el $74.4 \%$ al $51.6 \%$.

Así, probablemente la reducción de las tasas brutas de mortalidad se deba a una modificación en la composición etaria de los abandonados. Las cifras tienen una tendencia al descenso que confirman la admisión de un mayor porcentaje de niños con mayor edad, los que tenían más resistencia física ante las afecciones y que, a lo largo de estas décadas, se beneficiaron de las intervenciones institucionales, las mejoras en la alimentación y los tratamientos médicos.

\section{CuAdro n. ${ }^{\circ} 2$}

INGRESOS A LA CASA DE HUÉRFANOS 1898-1923

\begin{tabular}{|c|c|c|c|c|c|c|c|c|c|}
\hline Período & Entradas & Defunciones & $\begin{array}{c}\text { \% Mort. } \\
\text { Bruta }\end{array}$ & $\begin{array}{c}\text { Entradas } \\
<1 \text { año }\end{array}$ & $\begin{array}{c}\text { Defunciones } \\
<1 \text { año }\end{array}$ & $\begin{array}{c}\text { \% Mort. } \\
\text { Infantil }\end{array}$ & $\begin{array}{c}\text { \% Entradas } \\
<1 \text { año }\end{array}$ & $\begin{array}{c}\text { Entradas } \\
<1 \text { mes }\end{array}$ & $\begin{array}{c}\text { \% Entradas } \\
<1 \text { mes }\end{array}$ \\
\hline $1898-1902$ & 2.654 & 2.001 & 75.40 & 2.432 & 1.966 & 80.8 & 91.64 & 1.977 & 74.491 \\
\hline $1906-1910$ & 2.594 & 1.900 & 73.25 & 2.388 & 1.862 & 78.0 & 92.06 & 1.902 & 73.323 \\
\hline $1911-1915$ & 2.419 & 1.571 & 64.94 & 2.042 & 1.494 & 73.2 & 84.42 & 1.505 & 62.216 \\
\hline $1916-1920$ & 3.757 & 2.492 & 66.33 & 3.108 & 2.376 & 76.4 & 82.73 & 2.276 & 60.580 \\
\hline $1921-1923$ & 1.998 & 1.193 & 59.71 & 1.485 & 1.135 & 76.4 & 74.32 & 1.031 & 51.602 \\
\hline
\end{tabular}

Fuente: Libros de ingresos de los niños a la Casa de Huérfanos de Santiago.

\section{Las características de los niños ingresados a la Casa de Huérfanos}

La Casa de Huérfanos fue construida a finales del siglo XVIII en un terreno cedido por Manuel de Aguirre, localizado en la actual calle de Huérfanos. Esta zona corresponde al centro urbano de Santiago. Allí funcionó 
hasta 1855, cuando se trasladó a Providencia, zona rural que ofrecía amplios terrenos para la instalación del recinto. El edificio moderno se construyó durante la presidencia de Domingo Santa María (1885). ${ }^{29}$ Según el doctor Jorge Herrera, tuvo «23 grandes patios y permite aislar alrededor de mil niños, sin contar el personal de administración y los que se colocan en lactancia fuera del establecimiento». ${ }^{30}$ Dirigido por la Junta de Beneficencia, su servicio se encargó a las Hermanas de la Providencia. ${ }^{31}$ Comprendía secciones de expósitos o niños lactantes (menores de seis años, de los cuales algunos vivían con sus nodrizas) y la de huérfanos (mayores de seis años, denominada Casa, donde se educaban y enseñaban oficios). ${ }^{32}$

El reglamento de la Casa de Huérfanos, dictado el 10 de mayo de 1873, dispuso la admisión de los expósitos salidos de la lactancia, «manteniendo a los hombres hasta los diez años de edad en que deben abandonar la Casa, a escepcion de los sordos mudos i los necesarios para el servicio». ${ }^{33}$ El ingreso se realizó mediante el torno (un cubículo conectado al exterior del edificio donde se depositaba al menor), la entrega directa y la entrega policial. Sobre la primera, el doctor Luis Olivares Muñoz caracterizó su pésima condición sanitaria, producto de la ausencia de abrigo y una alimentación deficiente. ${ }^{34}$

29 La inauguración, noticiada por El Ferrocarril, fue encabezada por el presidente y sus ministros. Ahí explicó las funciones del recinto enmarcadas en un discurso caritativo. La institución recibía huérfanos para educarlos e instruirlos, devolviéndolos útiles y moralizados a la sociedad. Se proyectaba ampliar las capacidades de la Casa y habilitar una segunda sección para aceptar a los niños mayores de cinco años. La publicación destacaba la finalidad pedagógica del lugar: «los huérfanos no serán entregados a los que antes los solicitaban para aprovecharse de sus servicios, sino que serían amparados hasta que sean capaces de ganar su vida en las industrias que se les va a enseñar». «Inauguración de la Casa de Huérfanos», El Ferrocarril, Santiago, 2 de junio de 1885, 2.

30 Herrera, 1913, 65.

31 En 1853, las Hermanas de la Providencia arribaron a Chile desde Canadá. Durante su estadía en Valparaíso, el presidente Manuel Montt (1851-1861) les propuso que se hicieran cargo de los huérfanos de la capital. Para contextualizar el papel de las Hermanas de la Providencia en la historia contemporánea chilena, véase: Morín, 1899; Aliaga, 1993; Ponce de León, 2007, 60 y 61.

32 Los trabajos infantiles en talleres brindaron a los niños un oficio y, a futuro, un salario. En ese marco, el ministro de Marina sugirió al presidente de la Junta de Beneficencia crear una Escuela de Pesquería, ya que: «requería algunos de los niños de los Talleres de la Casa de Huérfanos (...) además de los niños de los talleres se podrían obtener algunos mui apropiados en la Escuela Correccional» (Sesiones de la Junta de Beneficencia, 13 de julio de 1907, f. 132). Por otro lado, Domingo Amunátegui, miembro de la Junta, proyectó el establecimiento de colonias agrícolas para los niños de la Casa de Huérfanos. Según él: «sería un medio de aprovechar la gran cantidad de niños en las faenas agrícolas del país y dejar espacio para el ingreso de nuevos pequeños en la institución» (Sesiones de la Junta de Beneficencia, 9 de diciembre de 1919, f. 564). Esta propuesta representa la imagen del niño trabajador construida desde las elites, que priorizaba el trabajo infantil sobre la escolarización.

33 Villarroel, 1906, 24.

34 Olivares, $1892,4$. 
Las formas de admisión a la Casa se basaron en los modelos antagónicos romano y alemán. El primero - implementado desde su fundaciónrecibía automática e incondicionalmente a los menores, favoreciendo en ellos la formación de una conciencia sin vínculos maternos. Para los especialistas, su debilidad radicó en la facilidad para aceptar párvulos llegados a sus puertas. El segundo condicionó el ingreso, mantuvo el vínculo padres-hijos y obstaculizó el abandono mediante tramitaciones burocráticas que, en ocasiones, pudieron favorecer el infanticidio. ${ }^{35}$

El abogado Julio Román comentó el debate moral francés sobre el uso del torno, un dispositivo central en el proceso de internación de menores. En este, mientras sus defensores apelaron a la disminución de la mortalidad de los niños sin familia, los detractores criticaron la promoción que daba al abandono de hijos legítimos e ilegítimos. ${ }^{36}$ Según ellos: «los padres indignos olvidaban sus deberes para con sus hijos, favoreciendo, el desorden i la inmoralidad». ${ }^{37}$ Algunos médicos propusieron mantener y mejorar los tornos agregándoles una campana que permitiera recoger a tiempo a los párvulos, impidiendo sus muertes.

Hacia 1894, había un torno en la calle Maestranza que conducía expósitos a la Casa de la Providencia. El sistema fue implementado allí por la considerable distancia entre el centro urbano y esa institución. A causa de esta situación, la Revista Médica de Chile opinó: «los más deplorables resultados, como fácilmente era de presumirlo, produjo el abandono de un gran número de niños en los alrededores de la casa donde existía el torno». ${ }^{38}$ La publicación denunció el restablecimiento del torno, el cual favorecía que «las madres sin entrañas abandonan para siempre a sus propios hijos a una suerte desconocida o a una muerte segura». ${ }^{39}$

El administrador de la Casa de Huérfanos señaló que el aumento de expósitos generaba mayores demandas de los servicios de una institución carente de recursos económicos para mantener, educar y enseñar algún oficio u ocupación honesta a los entrados, considerando que «en esta aflijente

35 Del Río y Commentz, 1909, 17.

36 Román Añibar, 1900, 52. Sobre la ilegitimidad en el período existen estudios médicos y jurídicos: Vargas, 1917; Matte, 1918; Arévalo, 1918; Letelier, 1918; Ipinza Besoain, 1920; Cordero Baños, 1923. Un estudio reciente que aborda los vínculos entre las tasas de ilegitimidad y la familia en Chile, es el de Díaz, Gallego y Lafortune, 2016, 37-79. Esta variable moral tuvo una comprobación en las estadísticas de la Casa de Huérfanos al fallecer los infantes clasificados como ilegítimos.

37 Román Añibar, 1900, 53.

38 Revista Médica de Chile, XXII, Santiago, 1894.

39 Idem. 
situación es de necesidad atender con preferencia al socorro de los huérfanos que se encuentran en la edad de la lactancia». ${ }^{40}$ Por los cuidados que requerían los recién nacidos y ante este colapso en el recinto, la Junta de Beneficencia invitaba a filántropos para hacerse cargo de algunos párvulos. En 1883, para mejorar la infraestructura del edificio, se llevaron a cabo trabajos para dotar de agua potable a la Casa, hasta entonces escasa. ${ }^{41}$

La mayoría de los niños internados en la Casa de Huérfanos falleció. Según el cuadro n. ${ }^{\circ} 3$, de los 15.315 infantes que entraron entre 1898-1923, murieron 10.503, lo que representa el 68,6\% del total. A juicio de los encargados de recibirlos, esto se produjo por las condiciones en que llegaban los párvulos, la mayoría enfermos. Aparentemente, las elevadas cifras de la mortalidad infantil de Santiago concordaban con las del recinto. De modo que el traslado de los infantes a la institución respondería más a circunstancias familiares (económicas, laborales, sanitarias) que a la motivación de intentar salvar la vida del recién nacido.

Por otro lado, el número de niños reclamados tras la internación, 2.132 de 15.315, refleja el tránsito de ellos entre los hogares y el establecimiento o bien la modificación de las situaciones que llevaron a su traslado. Cabe destacar las cifras de infantes que entraron y fueron criados hasta los seis años, cuando pasaron a la sección Casa de Huérfanos para aprender algún oficio.

\section{CUADRO N. ${ }^{\circ} 3$}

DESENLACE DE LOS NIÑOS INGRESADOS, 1898-1923

\begin{tabular}{|c|c|c|c|c|c|c|}
\hline Período & Murió & Reclamado & $\begin{array}{c}\text { Pasó a la Casa } \\
\text { de Huérfanos }\end{array}$ & Adoptado & $\begin{array}{c}\text { Se dio por } \\
\text { perdido }\end{array}$ & $\begin{array}{c}\text { No se } \\
\text { especifica }\end{array}$ \\
\hline $1898-1902$ & 2.060 & 190 & 437 & 53 & 14 & 0 \\
\hline $1906-1910$ & 2.385 & 408 & 327 & 44 & 32 & 4 \\
\hline $1911-1915$ & 2.334 & 559 & 409 & 51 & 43 & 94 \\
\hline $1916-1920$ & 2.510 & 584 & 427 & 45 & 6 & 220 \\
\hline $1921-1923$ & 1.214 & 389 & 198 & 30 & 0 & 209 \\
\hline Totales & 10.503 & 2.130 & 1.798 & 223 & 95 & 527 \\
\hline
\end{tabular}

Fuente: Libros de ingresos de niños a la Casa de Huérfanos de Santiago.

40 «Espósitos», El Independiente, Santiago, 9 de noviembre de 1866, 3.

41 «Casa de la Providencia», El Independiente, Santiago, 21 de febrero de 1883, 4. 
La mortalidad infantil en el establecimiento fue objeto de debate entre los médicos, pues era el único espacio responsable de aceptar niños sin hogar. Asimismo, hasta fines del siglo XIX, funcionó como espacio de socorro a los enfermos, pues no existía un hospital infantil y los dispensarios atendían solamente de manera ambulatoria. Sin embargo, el recinto no respondía a tales propósitos. A ello se agregaba el aumento constante en la demanda de los servicios, lo que ocasionó el deterioro de la higiene del lugar, la escasez de atenciones médicas y la falta de recursos.

El cuadro n. ${ }^{\circ} 4$ recoge la edad de entrada de los niños a la Casa. Claramente, la mayoría de los ingresos se efectuó durante la segunda semana de vida, lo que representaba el 33,3\% del total de 13.430 fichas que indicaron el momento de la internación. Es posible suponer que este período respondió a la recuperación de la madre tras el parto, a su decisión de entregar el párvulo y a la realización de los trámites de rigor. Igualmente, el primer mes de vida fue el período que tuvo 8.691 párvulos trasladados y que equivale al $64,7 \%$ de la muestra. Justo, esta época era crítica para la sobrevivencia de un recién nacido, pues se encontraba más vulnerable a las condiciones del medio y necesitaba mayores preocupaciones, tanto en el exterior como en el régimen de internación. Al progresar en segmentos de edad, se aprecia una disminución de niños ingresados y un aumento del período de sobrevivencia (sobre todo después del año).

Los médicos vincularon la alta mortalidad con la condición agónica que muchos de ellos presentaban al ingresar. Ante ese estado de salud, varios padres prefirieron entregarlos a la Casa antes que costear el entierro y realizar los trámites laicos o eclesiásticos. Un informe enviado por el Consejo de Defensa Fiscal al Ministerio de Justicia expuso que «el inspector del Rejistro Civil señala a V.S. que algunos párrocos de lugares en donde solo existen cementerios parroquiales se niegan a enterrar en ellos a las criaturas que nacen muertas. El señor inspector desea saber si esos curas tienen derecho para proceder así, o si están obligados a efectuar esas sepultaciones en vista del pase que espida el oficial civil de la localidad». ${ }^{42}$

Se puede presumir que un número importante de los menores que ingresaban en las primeras semanas presentaban algún tipo de complicación médica, lo cual explicaría los altos índices de mortalidad en la Casa

42 Oficio enviado del Consejo de Defensa Fiscal al Ministerio de Justicia que aparece fechado el 12 de diciembre de 1903 y publicado en la prensa. «Sepultación de párvulos», Las Últimas Noticias, Santiago, 14 de diciembre de 1903, 5 . 
PABLO CHÁVEZ ZÚÑIGA Y JOSÉ JULIÁN SOTO LARA

\begin{tabular}{|c|c|c|c|c|c|c|c|}
\hline \multirow{16}{*}{ 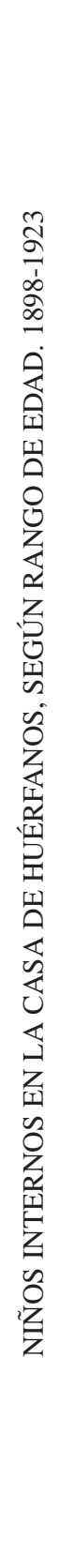 } & $\mid \begin{array}{ll}\tilde{z} & \tilde{y} \\
\tilde{z} & \vdots \\
z & \vdots \\
z & 1\end{array}$ & లి & 0 & 0 & 0 & $\stackrel{0}{ }$ & o \\
\hline & $\hat{b}=$ & $\stackrel{\infty}{\infty}$ & - & $m$ & $n$ & $\stackrel{0}{=}$ & $\mathscr{\vartheta}$ \\
\hline & 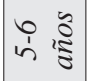 & $\vec{\sim}$ & $\mathrm{N}$ & $\forall$ & $\stackrel{\sim}{\sim}$ & $\vec{n}$ & t \\
\hline & $\begin{array}{ll}n & \tilde{g} \\
\dot{\gamma} & \stackrel{\Xi}{\Xi}\end{array}$ & 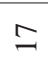 & $\cong$ & $\stackrel{\infty}{m}$ & 으 & $\infty$ & $\sqrt{n}$ \\
\hline & 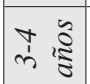 & $\stackrel{\curvearrowright}{ }$ & mे & $+\infty$ & 戸 & ส & $\underset{\infty}{\infty}$ \\
\hline & 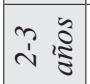 & $n$ & $\hat{n}$ & হ & ชิ & $\hat{0}$ & $\stackrel{\bullet}{\stackrel{十}{\sigma}}$ \\
\hline & $\begin{array}{cc}\sim & \tilde{E} \\
& \stackrel{\Xi}{\Xi}\end{array}$ & $\stackrel{N}{N}$ & 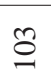 & g & $\stackrel{\Delta}{\sim}$ & $\stackrel{g}{\exists}$ & $\bar{\infty}$ \\
\hline & 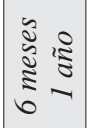 & 으 & 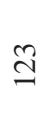 & $\cong$ & ஜ & $\underset{\Xi}{ \pm}$ & s \\
\hline & $\begin{array}{ll}0 & \vdots \\
\cdots & \vdots \\
& \Xi\end{array}$ & さે & $\stackrel{\infty}{=}$ & 옴 & $\frac{\sim}{d}$ & ปี & $\vec{N}$ \\
\hline & 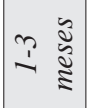 & $\vec{\sim}$ & $\stackrel{n}{\sim}$ & 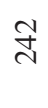 & $\stackrel{+}{\Im}$ & $\stackrel{\infty}{\stackrel{\sim}{\circ}}$ & $\begin{array}{l}0 \\
m \\
m\end{array}$ \\
\hline & 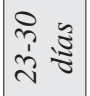 & nิ & $\stackrel{8}{8}$ & g) & $\hat{\sim}$ & $\vec{m}$ & $\begin{array}{l}\circ \\
\infty\end{array}$ \\
\hline & 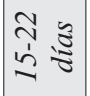 & 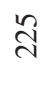 & 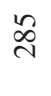 & ì & $\overrightarrow{0}$ & $\underset{\infty}{ \pm}$ & ָָ \\
\hline & 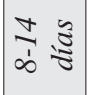 & $\stackrel{\circ}{=}$ & $\ddot{\circ}$ & $\stackrel{0}{1}$ & 党 & $\tilde{\hat{n}}$ & $\begin{array}{l}\stackrel{0}{f} \\
\stackrel{+}{+}\end{array}$ \\
\hline & 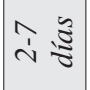 & $\stackrel{\sim}{\infty}$ & $\stackrel{\infty}{\infty}$ & ঐे & 离 & $n$ & 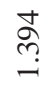 \\
\hline & $\overline{0}: \mathbb{Z}$ & $\stackrel{\circ}{2}$ & ฮิ & $\stackrel{9}{=}$ & $\stackrel{\infty}{ \pm}$ & $\stackrel{\infty}{m}$ & $\hat{\rho}$ \\
\hline & $\begin{array}{c}5 \\
0 \\
0 \\
0 \\
0\end{array}$ & 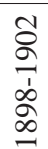 & $\begin{array}{l}\frac{0}{2} \\
\frac{1}{2} \\
\frac{1}{2}\end{array}$ & $\begin{array}{l}\frac{n}{a} \\
\frac{1}{2} \\
\frac{a}{2}\end{array}$ & 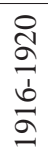 & $\frac{\widetilde{a}}{\stackrel{a}{a}}$ & $\frac{0}{\tilde{J}}$ \\
\hline
\end{tabular}


CuAdro n. ${ }^{\circ} 5$

\section{RELACIÓN ENTRE NIÑOS INGRESADOS ENFERMOS Y FALLECIMIENTOS}

\begin{tabular}{|c|c|c|}
\hline Año & Enfermos & Fallecieron \\
\hline 1898 & 289 & 228 \\
\hline 1899 & 412 & 339 \\
\hline 1900 & 321 & 274 \\
\hline 1901 & 362 & 286 \\
\hline 1902 & 253 & 210 \\
\hline 1906 & 199 & 172 \\
\hline 1907 & 151 & 134 \\
\hline 1908 & 214 & 191 \\
\hline 1910 & 288 & 221 \\
\hline 1911 & 123 & 105 \\
\hline
\end{tabular}

Fuente: Libros de ingresos de niños a la Casa de Huérfanos de Santiago.

de Huérfanos..$^{43}$ El doctor Jenaro Lisboa criticó «una condición irregular i precaria [con] que vienen al mundo estos seres desventajados dados a luz de ordinario clandestinamente en las más funestas condiciones; todo hace que lleguen a la Casa a morir una vez sobre los tres a lo menos». ${ }^{44} \mathrm{En}$ su opinión, los problemas del servicio médico se debían tanto a la escasez de recursos como a la resistencia popular para usar medicamentos, pues se pensaba que los remedios causaban las enfermedades. Lisboa sabía las limitantes de aquellos, porque «ha de curarse el niño en las más adversas condiciones, pues ha de volver con su nodriza al pobre hogar de ésta, a habitaciones pobres e infestas i no pocas veces en medio de una criminal indolencia». ${ }^{45}$ Por su parte, el doctor Jorge Herrera comprendió que el establecimiento era insuficiente para todos los niños ingresados «cuyo exceso perjudica la salubridad de los mismos». ${ }^{46}$

43 El porcentaje de niños consignados como enfermos en los ingresos durante algunos años fue el siguiente: $1898(82,33 \%), 1899(68,78 \%), 1900(51,27 \%), 1901(56,04 \%), 1902(47,65 \%)$, $1906(44,02 \%), 1907(19,81 \%), 1908(31,1 \%), 1910(43,5 \%)$ y $1911(17,06 \%)$. Cabe señalar que este dato fue anotado de manera esporádica ya que se encuentra concentrado en algunos años y en meses determinados.

44 Lisboa, 1886, 16.

45 Lisboa, $1886,18$.

46 Herrera, 1913, 66. 
Según la información consignada en el cuadro n. ${ }^{\circ}$ 5, la mayoría de los niños que ingresaron enfermos a la Casa fallecieron. Las anotaciones de las religiosas se limitaron a constatar esto, pero no definieron a qué tipo de malestar correspondía, ni realizaron una descripción de las afecciones. Probablemente, esta ausencia de los diagnósticos o cualquier referencia a elementos médicos se genera por la clase de registro elaborada y la forma de recolectar información. Al no existir una especialización pediátrica, la calificación de enfermos fue atribuida a partir de la observación visual.

\section{La ilegitimidad}

La ilegitimidad del nacimiento estuvo dada por la ausencia del vínculo matrimonial entre los procreadores del nacido. Para el Derecho representó algo irregular que infringía el orden legal establecido y que incumplía con los parámetros instaurados por la Iglesia hasta 1883 y, desde esa fecha, por el Estado. Bajo esa línea moral, para doctores y abogados, los niños que nacían de uniones temporales no recibirían los cuidados necesarios y la madre, al regresar a sus obligaciones laborales, lo abandonaría. De ahí el interés de los médicos y la élite política por constituir un modelo familiar y excluir a los distanciados de esos cánones. Las diversas características que le asignaron a este segmento de la infancia - pobre, enferma, heredera de padres alcohólicos- reflejan la construcción de causalidades de mortalidad unos días después de los nacimientos.

El cuadro n. ${ }^{\circ} 6$ expresa que los niños ilegítimos internados en la institución fueron más numerosos que los legítimos. Las cifras de ilegítimos confirmaban que las familias sin matrimonios legalizados recurrieron en mayor medida a los servicios de la Casa. Las madres enviaron a sus hijos para que recibieran los cuidados alimentarios necesarios para su crecimiento. Los registros eclesiásticos constaron la situación matrimonial de los padres, puesto que de ese dato infirieron la precariedad de los infantes. En esos años, las ciencias y las élites políticas elaboraron un discurso moral familiar que relacionaba la disolución del grupo con la falta de cuidados y la mortalidad de los recién nacidos.

El cuadro n. ${ }^{\circ} 6$ confirma que los niños ilegítimos fueron el grupo con mayor índice de mortalidad en la institución. De los 10.632 ingresados, 7.939 fallecieron, lo que representa el 74,6 \% de la muestra. Con este dato, 
los médicos, la Iglesia y los políticos reforzaron la asociación entre ilegitimidad y mortalidad. Las perspectivas señaladas se basaron en este punto para desarrollar las explicaciones de la mortalidad infantil. En esta visión subyació la lógica del descuido, una idea que privilegió la moralidad sobre otros elementos como la alimentación descompuesta, el contagio de enfermedades o por falta de aislamiento.

\section{CuAdro N. ${ }^{\circ} 6$}

RELACIÓN DE INGRESOS, LEGITIMIDAD-ILEGITIMIDAD Y MORTALIDAD

\begin{tabular}{|l|c|c|c|}
\hline \multicolumn{1}{|c|}{ Categoría } & Entradas & Defunción & \% mortalidad \\
\hline Legítimos & 3.655 & 1.946 & 53.24 \\
\hline Ilegítimos & 10.632 & 7.939 & 74.67 \\
\hline No especificada & 950 & 585 & 61.58 \\
\hline Otros $^{47}$ & 49 & 33 & 67.35 \\
\hline
\end{tabular}

Fuente: Libros de ingresos de niños a la Casa de Huérfanos de Santiago.

Hasta fines del siglo XIX, los médicos y políticos consideraban la mortalidad infantil como un problema social, diagnóstico reñido con la precaria capacidad para solucionarlo. En ese período, la medicina infantil no era considerada como una profesión hospitalaria, porque no existían lugares especializados para el tratamiento de niños y los hospitales eran instituciones benéficas.

En líneas generales, la elaboración de perfiles de los infantes internados configuró un modelo de familia que respondió al paradigma cultural del período. $\mathrm{Al}$ amparo de este diagnóstico, médicos y políticos justificaron una crítica dirigida a amplios sectores poblacionales con los argumentos que hemos señalado. Ese análisis se tradujo en un Estado que miraba a los habitantes como responsables de sus malestares y que respondía, mediante la beneficencia, a las necesidades de las personas. A través de la Casa entregaba a los infantes los cuidados no prodigados por sus familiares.

47 La categoría «otros» en los cuadros $n .^{\circ} 5$ y n. ${ }^{\circ} 6$ se refiere a los registros de niños con padres desaparecidos o desconocidos. 


\section{Discursos sobre el abandono}

La Casa de Huérfanos funcionó como un lugar de circulación de los infantes y punto de conexión entre los tribunales, la policía y las familias. Los niños, en ocasiones, fueron reclamados y nuevamente ingresados. En esta perspectiva, el establecimiento cumplió el papel de recibir y entregar niños a otras familias con madres asalariadas por tal labor, considerando esa agencia femenina como un apoyo al funcionamiento de la organización.

La toma de decisión para internar niños fue variada. Hubo familias que ingresaron varios hermanos, otras que escogieron solo a uno de ellos, y otras que, en casos excepcionales, decidieron entregar a un gemelo para que el otro se criara en la familia. ${ }^{48}$ Por ejemplo, Margarita Orta, dijo que «por verse abandonada de su marido y con dos hijos más, dejaba abandonada a ésta hija». ${ }^{49}$ Otra madre, que dijo carecer de recursos económicos, internó a su hija prometiendo su reclamo, pero la criatura falleció días después. ${ }^{50}$

Probablemente, la Casa de Huérfanos era vista como un escenario para el cuidado infantil. Según las fuentes, hubo una presencia considerable de hermanos internados ( 270 casos entre dos y cinco niños), como, por ejemplo, Petronila de 16 días, hermana de Juan de Dios (5 años), José (4 años) y Rogelio (8 años), respectivamente. En este caso, si bien no se especifican los motivos del ingreso, los cuatro entraron en octubre de 1899. ${ }^{51}$ Tal fue la situación de los hermanos Raúl y Lidia Vidal, ambos de 17 días, ingresados luego de la muerte de su madre durante el parto y bajo la promesa de «reclamarlos pagando su crianza». ${ }^{52}$ Luego de tres meses, solo el pequeño Raúl fue reclamado y Lidia siguió en la sección de lactancia hasta que cumplió los seis años y en 1923 pasó a la formación en talleres.

La ausencia paterna en la familia condicionó el traslado de menores a la Casa. María Castro, por ejemplo, renunció a su hijo por tener otros

48 Ingreso n. ${ }^{\circ} 10.728(11 / 05 / 1915)$.

49 Entre las madres que debieron elegir un hijo entre varios para que ingresara al recinto, véase los ingresos: n. ${ }^{\circ} 1.400$ (11/03/1902), n.. 13.478 (25/04/1919), n. ${ }^{\circ} 13.898$ (18/10/1919).

50 Ingreso n. $^{\circ} 11.730(22 / 10 / 1916)$.

51 Ingreso n..$^{\circ} 14.289$ (04/10/1899), n. ${ }^{\circ} 14.319$ (18/10/1899), n. ${ }^{\circ} 14.320(18 / 10 / 1899)$, n. ${ }^{\circ} 14.322(19 / 10 / 1899)$.

52 Ingreso n. ${ }^{\circ} 11.735(24 / 10 / 1916)$, n..$^{\circ} 11.736(24 / 10 / 1916)$. 
cuatro y haber sido abandonada por su marido. ${ }^{53}$ Laura Moraga, por su lado, entregó a su hijo porque su marido contrajo matrimonio «con otra y así no pudo mantener al chico». ${ }^{54}$

El crecimiento del mercado laboral y la precariedad favorecieron la búsqueda femenina de trabajo, ${ }^{55}$ pero también fundamentaron en ellas la decisión del abandono infantil. ${ }^{56}$ Destacamos el discurso moral de la elite médica y política hacia la supuesta holgazanería, desempleo y criminalidad popular que aumentaba las probabilidades del abandono infantil. ${ }^{57}$ Una parte del pueblo, en la práctica, utilizó la Casa de Huérfanos como una residencia transitoria para sus hijos.

Este proceso generó situaciones que conformaron elementos de reproducción social y consolidación de las desigualdades ${ }^{58}$ Para las mujeres, conseguir y mantener un trabajo durante la maternidad conllevó dificultades. Así lo demuestran algunas declaraciones: «dejó a su hijo por no poder emplearse con él» ${ }^{59} \mathrm{o}$ «su madre lo deja porque es pobre y no la admiten con el niño para servir». ${ }^{60}$ Otros relatos atribuyeron complicaciones para emplearse, como lo expresó Rosa Díaz, quien dejó a su retoño, puesto que «se va a emplear con una hermana del chico, pero no la reciben con los dos». ${ }^{61}$

53 Ingreso n. ${ }^{\circ} 1.464(26 / 04 / 1902)$.

54 Ingreso n. ${ }^{\circ} 11.993(12 / 03 / 1917)$.

55 En los censos y anuarios estadísticos es posible rastrear la composición del trabajo femenino en Chile. Una investigación de Elisabeth Quay Hutchison analizó la alta inserción de las mujeres en el mercado laboral en la segunda mitad del siglo XIX, que se reduciría comenzando el XX. La autora explicó ese cambio con el perfeccionamiento metodológico en la recolección de datos y la confección de la estadística, así como por la distorsión categorial entre los instrumentos usados en ambos tiempos: la mujer dedicada a labores inestables era consignada parte de la masa laboral, lo que cambió en los censos y pasó a ser considerada como dedicada a las «labores de su sexo». Véase: Quay Hutchison, 2000, 417-434.

56 A modo de referencia, la pobreza y búsqueda de empleo fue argumento para el ingreso de niños al establecimiento en las siguientes anotaciones: . $^{\circ} 13.598,(15 / 08 / 1898)$, n. $^{\circ} 13.755(04 / 11 / 1898)$, n. ${ }^{\circ} 9.019$ (31/10/1912), n. ${ }^{\circ} 9.455$ (07/07/1913), n. ${ }^{\circ} 9.800(13 / 12 / 1913)$, n. ${ }^{\circ} 10.524(10 / 01 / 1915)$, n. ${ }^{\circ} 13.462(21 / 04 / 1919)$, n. $^{\circ} 13.518$ (13/05/1919), n. ${ }^{\circ} 13.520(13 / 05 / 1919),{ }^{\circ} 13.716(05 / 08 / 1919)$, n. ${ }^{\circ} 13.795$ (12/09/1919), n. ${ }^{\circ} 13.845$ (29/09/1919), n. ${ }^{\circ} 13.860(04 / 10 / 1919),{ }^{\circ}{ }^{\circ} 13.931(02 / 11 / 1919)$, n. ${ }^{\circ} 13.947$ (09/11/1919), n. ${ }^{\circ} 13.986$ (20/11/1919), n. ${ }^{\circ} 14.080(27 / 12 / 1919)$, n. $^{\circ} 14.097(03 / 01 / 1920)$, n. ${ }^{\circ} 14.141$ (21/01/1920), n. ${ }^{\circ} 14.193(13 / 02 / 1920)$, n. $^{\circ} 14.195(16 / 02 / 1920)$, n. $^{\circ} 14.202(19 / 02 / 1920)$, n. ${ }^{\circ} 14.379(24 / 05 / 1920)$, n. $^{\circ} 14.501(14 / 07 / 1920)$.

57 Del Castillo Troncoso, 2009. Para Michel Foucault, el problema del Estado, en el siglo XIX, era fijar a los obreros al aparato productivo, de someterlos a un ritmo fijo e imponerles una disciplina laboral. Foucault, 1996, 48.

58 Milanich, 2011, 29-62.

59 Ingreso $n .^{\circ} 10.861(24 / 07 / 1915)$, n. $^{\circ} 12.876(15 / 07 / 1918)$

60 Ingreso n. ${ }^{\circ} 11.964(21 / 02 / 1917)$.

61 Ingreso n. ${ }^{\circ} 14.473(03 / 07 / 1920)$. 
Otra circunstancia del ingreso de niños en la Casa fue la alta mortalidad materna (576 ocasiones). En esta época, el parto representaba una probabilidad de muerte debido a una obstetricia incipiente y una higiene hospitalaria y de los hogares de las parteras deficiente. ${ }^{62}$ Los neonatos huérfanos de madre tuvieron igual destino (440 niños muertos, correspondiente al $76 \%$ de la muestra). Un episodio particularmente complejo fue la muerte de Rosa Gutiérrez en el Hospital San Borja luego de parir a las trillizas María Carolina, María Julia y María Elena, las que en la Casa no sobrevivieron un mes. ${ }^{63}$

En ocasiones, cuando las mujeres enfermaban a causa del parto u otras dolencias (tuberculosis ${ }^{64}$ ) eran ingresadas en el Lazareto y sus hijos en la Casa, rotulados como «niño de hospital», ${ }^{65}$ a la espera de ser reclamados por sus madres. En ocasiones, las madres no volvían por sus hijos. Rufina Bustamante estuvo internada desde el 23 de mayo de 1900, dejando al menor Alfredo Pavez, de 3 años, como niño de hospital; meses después, desde el Hospital San Borja avisaron que «la madre se había ido dejando a su hijo»; ${ }^{66}$ posteriormente, el 3 de enero de 1901 recibió el bautismo. Las últimas noticias del menor corresponden al día 2 de julio de 1903 cuando, con 6 años, pasó a la sección Casa del establecimiento.

El número de ingresos tras la muerte o enfermedad de la madre generó discusiones en la Junta de Beneficencia. El doctor Roberto del Río propuso la conformación de una sección receptora de «todas las guaguas, hijos de madres enfermas y que sean atendidas en los hospitales o fallecidas en

62 La historización del parto fue realizada por Soledad Zárate, quien estudió las relaciones de género que vincularon a parturientas, parteras, matronas y médicos. Además, analizó el quiebre y las trayectorias producidas entre los oficios de las parteras y el surgimiento de la obstetricia. Sus descripciones permiten comprender las dificultades en las historias de mujeres que explican la mortalidad durante el parto y el puerperio (Zárate, 2007). A comienzos del siglo veinte aparecieron escritos los cuidados de las madres como factor que contribuiría a disminuir la mortalidad infantil. Recomendamos el trabajo de Korner, 1913.

63 Ingreso n. ${ }^{\circ} 13.994$ (21/11/1919), n. ${ }^{\circ} 13.995$ (21/11/1919), n. ${ }^{\circ} 13.996(21 / 11 / 1919)$.

64 Ingreso n. ${ }^{\circ} 13.899(18 / 10 / 1919),{ }^{\circ}{ }^{\circ} 14.365(19 / 05 / 1920)$.

65 Casos en que los niños pasaron desde el Hospital (por la enfermedad o muerte de la madre) a la Casa de Huérfanos, véase ingresos n. ${ }^{\circ} 13.883$ (12/01/1899), n. ${ }^{\circ} 13.900$ (19/01/1899), n. ${ }^{\circ} 1.468$ (30/04/1902), n. ${ }^{\circ} 1.474$ (04/05/1902), n. ${ }^{\circ} 7.300$ (09/07/1910), n. ${ }^{\circ} 7.517(08 / 10 / 1910)$, n. $^{\circ} 7.780$ (22/02/1911), n. ${ }^{\circ} 7.884(01 / 05 / 1911)$, n. $^{\circ} 7.890(07 / 05 / 1911)$, n. $^{\circ} 8.334(30 / 11 / 1911)$, n. $^{\circ} 8.429$ (10/01/1912), n. ${ }^{\circ} 8.797$ (29/07/1912), n. ${ }^{\circ} 10.479$ (21/12/1914), n. ${ }^{\circ} 10.837$ (12/07/1915), n. ${ }^{\circ} 10.877$ (01/08/1915), n. ${ }^{\circ} 10.961$ (15/09/1915), n. ${ }^{\circ} 11.007(04 / 10 / 1915)$, n. $^{\circ} 11.137(03 / 12 / 1915)$, n. $^{\circ} 11.907$ (20/01/1917), n. ${ }^{\circ} 11.968$ (23/02/1917), n. ${ }^{\circ} 12.899$ (22/07/1918), n. ${ }^{\circ} 13.365(04 / 02 / 1919)$, n. $^{\circ} 13.612$ (26/06/1919), n. ${ }^{\circ} 16.057(09 / 08 / 1922)$, n. ${ }^{\circ} 16.494(21 / 01 / 1923)$.

66 Ingreso n. $320(05 / 08 / 1900)$. 
ellos». ${ }^{67}$ Preocupado por la situación infantil, agregaba que se estaban produciendo rechazos de neonatos. En respuesta, el subadministrador expuso que el recinto estaba lleno (1.050 niños en la sección lactancia y 192 en el pesebre) y que «siendo su objeto recibir los espósitos, no puede destinar sus dependencias a niños que tienen madre, se han estado recibiendo por escepcion y en vista de la gran cantidad de enfermos que hay con motivo de la epidemia». ${ }^{68}$ Finalmente, la administración habilitó una sección de tránsito, «para evitar el rechazo de niños que no siendo huérfanos necesitan un albergue transitorio». ${ }^{69} \mathrm{La}$ Asistencia Pública resguardó a los menores remitidos en la Casa de Huérfanos, estableciendo que se apuntase en la libreta de admisión de la madre enferma una indicación para que no se le concediera el alta mientras no resolviera la situación de su hijo.

Cuando la madre o el padre eran interdictos, ${ }^{70}$ la internación de sus hijos en la Casa de Huérfanos era común. Por eso hallamos niños remitidos con una carta explicitando que son hijos de insanos ${ }^{71}$ o descripciones señalando que «su madre es una pobre demente incapaz de cuidar al chico», ${ }^{72} \ll$ la madre es inválida y viciosa» ${ }^{73} \mathrm{o}$ «la madre es una mujer loca que no sabe decir su nombre». ${ }^{74}$ Por ejemplo, tras la interdicción de Margarita Servet, su hijo de 9 años fue calificado de «tonto» en su ficha de entrada. ${ }^{75}$ Los hombres fueron mencionados escasamente por este motivo. En esta época, una mujer sorda, muda o ciega era considera una enferma, condición

67 Sesiones de la Junta de Beneficencia, 2 de diciembre de 1919, f. 561-562.

68 Sesiones de la Junta de Beneficencia, 2 de diciembre de 1919, f. 562.

69 Sesiones de la Junta de Beneficencia, 9 de diciembre de 1919, f. 563.

70 Consiste en el acto jurídico que establece la declaración legal de incapacidad de una persona para administrar sus bienes. A pesar de que el Código Civil chileno no define las enajenaciones mentales, en el artículo 456 y siguientes de este cuerpo legal está tipificado que los jueces tomarán estas resoluciones según informe y examen de los médicos.

71 Entre los ingresos que describieron la internación de la madre en la Casa de Orates, encontramos: n. ${ }^{\circ} 13.845$ (17/12/1898), n. ${ }^{\circ} 5.227$ (22/06/1907), n..$^{\circ} 5.490(08 / 10 / 1907)$, n. $^{\circ} 5.861$ (25/04/1908), n. ${ }^{\circ} 6.267$ (14/11/1908), n. ${ }^{\circ} 7.327$ (19/07/1910), n. ${ }^{\circ} 7.591$ (18/11/1910), n. ${ }^{\circ} 7.718$ (14/01/1911), n. 7.739 (29/01/1911), n. 8.218 (14/10/1911), n. ${ }^{\circ} 9.004(25 / 10 / 1912)$, n. $^{\circ} 10.619$ (06/03/1915), n. ${ }^{\circ} 11.234(20 / 01 / 1916)$, n. $^{\circ} 11.435$ (07/06/1916), n. ${ }^{\circ} 11.877(07 / 01 / 1917)$, n. $^{\circ} 13.453$ (12/04/1919), n. ${ }^{\circ} 13.455$ (14/04/1919), n. ${ }^{\circ} 13.622$ (02/07/1919), n. ${ }^{\circ} 13.771(06 / 09 / 1919)$, n. $^{\circ} 14.026$ (03/12/1919), n. ${ }^{\circ} 14.149$ (24/01/1920), n. ${ }^{\circ} 14.254$ (18/03/1920), n. ${ }^{\circ} 14.433(16 / 06 / 1920)$, n. $^{\circ} 14.670$ (12/09/1920), n. ${ }^{\circ} 14.869$ (23/11/1920), n. ${ }^{\circ} 15.076(21 / 02 / 1921)$, n. $^{\circ} 15.517(15 / 10 / 1921)$, n. $^{\circ} 15.599$ (21/11/1921), n. ${ }^{\circ} 15.940$ (08/06/1922), n. ${ }^{\circ} 15.992$ (06/07/1922). Para profundizar en el estudio histórico de la locura, véase las investigaciones de Correa, 2012, 88-109; 2013.

$\begin{array}{ll}72 & \text { Ingreso n. }{ }^{\circ} 13.618(29 / 06 / 1919) . \\ 73 & \text { Ingreso n. }{ }^{\circ} 15.883(06 / 05 / 1922) . \\ 74 & \text { Ingreso n. }{ }^{\circ} 16.868(05 / 07 / 1923) . \\ 75 & \text { Ingreso n. }{ }^{\circ} 849(03 / 06 / 1901) .\end{array}$ 
que argumentó el abandono. ${ }^{76} \mathrm{La}$ incapacidad física de los progenitores reducían las posibilidades de obtener sustento económico, dando paso a precariedades en el grupo familiar; por ejemplo, una anotación indicaba «el padre es ciego». ${ }^{77}$ Estas condiciones se agravaban cuando la madre tenía muchos niños, escasez de recursos y un marido inválido. ${ }^{78}$

\section{Los cuidados en la infancia}

La Casa de Huérfanos contrató a nodrizas para que se encargaran del cuidado y alimentación de los niños. La función de las nodrizas en la Casa de Huérfanos tuvo partidarios y detractores. Los primeros acusaron su escasez, ya que ellas preferían los trabajos particulares con mejores salarios. Los segundos las calificaron de «mercenarias», reemplazables por leche de vaca o burra. ${ }^{79}$ Esta discusión se dio en medio de la alta mortalidad - al interior de la Casa - producida por enfermedades estomacales explicadas por la alimentación inapropiada de carácter mixto (mezcla entre leche natural y artificial). ${ }^{80}$ Los médicos concordaban en que el aumento de niños muertos en verano se debía, entre otras razones, a la descomposición de la leche consumida. ${ }^{81}$ Esta circunstancia nos permite interpretar que se utilizaba más leche artificial que materna.

En la crítica hacia las nodrizas subyació la concepción tradicional de la familia. Supuestamente, los padres desinteresados de sus hijos recurrían a ellas. Asimismo, el papel que cumplían, y por el que recibían un salario, representaba una alternativa a la lactancia materna que intentaba implantar la medicina. Sin embargo, la práctica en ocasiones distó del discurso moralizante, pues hubieron nodrizas que crearon vínculos maternales con los huérfanos y cedieron sus apellidos para las criaturas. ${ }^{82}$ Otros huérfanos

76 Menciones a discapacidades de la madre en los siguientes ingresos: n. ${ }^{\circ} 10.032(06 / 05 / 1914)$, n. ${ }^{\circ} 10.129(07 / 07 / 1914)$, n. $^{\circ} 14.257$ (18/03/1920), n. ${ }^{\circ} 15.315$ (13/07/1921), n. ${ }^{\circ} 15.791$ (13/03/1922).

77 Ingreso n. ${ }^{\circ} 10.991(26 / 09 / 1915)$.

78 Registros en que la invalidez del hombre era causa de ingreso de un infante al recinto: n. ${ }^{\circ} 12.431(23 / 10 / 1917)$, n. $^{\circ} 12.471(09 / 11 / 1917)$, n. $^{\circ} 12.968(17 / 08 / 1918)$.

79 Olivares, $1892,7$.

80 Para investigar la alimentación de los lactantes, véase: Calvo Mackenna, 1913; Sánchez Cruz, 1913; Pulido Illanes, 1913; Simon, 1913.

81 Entre 1898 y 1923 la cantidad de niños fallecidos por mes fue: enero (1.282 niños), febrero (837), marzo (657), abril (628), mayo (710), junio (695), julio (690), agosto (818), septiembre (815), octubre (874), noviembre (1.117) y diciembre (1.380).

82 Registros en los que aparece la nodriza otorgando su apellido al menor que cuidaba: n. ${ }^{\circ} 10.910(20 / 08 / 1915)$, n. $^{o} 11.032(16 / 10 / 1915)$, n. $^{\circ} 11.620(01 / 09 / 1916)$. 
fueron adoptados por ellas, ${ }^{83}$ como el caso de Clara de Asís Reyes, abandonada en Providencia. ${ }^{84}$ Suerte distinta tuvo la niña Uberlinda, quien sufrió «la muerte de su madre hace tres años y ahora murió su nodriza», ${ }^{85}$ debiendo reingresar a la Casa de Huérfanos.

Algunas nodrizas vivieron lejos de la Casa, trasladándose allí mensualmente para recibir sus salarios e inspeccionar a los párvulos. A pesar que era obligatorio «presentar al niño y recurrir a los médicos de la Casa cuando enfermaran», ${ }^{86}$ esto se incumplió. El doctor Olivares propuso medidas para modificar esta situación: primero, que los médicos practicaran una inspección de los niños cuidados por nodrizas; segundo, que el establecimiento entregase una gratificación a las mujeres que devolvieran sano al menor dado para criar; tercero, proporcionar a cada nodriza una cartilla con los síntomas principales y los tratamientos de las enfermedades más frecuentes durante los primeros meses de nacidos, recomendando la asistencia médica apenas se desarrollase alguna dolencia grave. Esto último las dotó de un rol sanitario activo, desmontando el temor a los hospitales como espacios premortales y difundiendo los progresos pediátricos.

Cuando la adopción de niños realizada por alguna familia fracasaba, aquellos eran trasladados a la Casa. La violación, por parte de las familias, de los acuerdos económicos legitimó la función de la institución. Así se desprende de las declaraciones de Clara Reyes, Raquel Guajardo y Sara López, quienes se sorprendieron ante la desaparición de las progenitoras. ${ }^{87}$ También hubo encargados que esperaron años y sin éxito el retorno de las madres. A propósito, María González crió por cuatro años a un niño ajeno, pues «la madre lo dejó en la casa cuando guagua y no volvió por él» ${ }^{88} \mathrm{El}$ incumplimiento de los acuerdos también fue ocasionado por las muertes de las madres. ${ }^{89}$

83 En escasas oportunidades, la relación entre niño-nodriza derivó hacia el maltrato infantil. Una excepción fue publicada en «Cadáver de un párvulo», El Ferrocarril, Santiago, 25 de octubre de 1885 , 2. La noticia narró la muerte de una niña que presentaba «contusiones, heridas en el cuello y a un lado del pecho». Fue puesta a disposición del Juez del Crimen.

84 Ingreso n. ${ }^{\circ} 11.181(29 / 12 / 1915)$.

85 Ingreso n. $^{\circ} 11.252(03 / 02 / 1916)$. En este registro solo aparece el nombre de la niña.

86 Olivares, $1892,7$.

87 Ejemplos de la situación en que la madre acordó la crianza del hijo o lo dejó con una persona, pero no regresó por múltiples circunstancias: ingreso n. ${ }^{\circ} 271$ (11/07/1900), n. ${ }^{\circ} 7.777$ (20/02/1911), n. ${ }^{\circ} 7.933(02 / 06 / 1911)$, n. $^{\circ} 8.122(31 / 08 / 1911)$, n. $^{\circ} 11.054(28 / 10 / 1915)$, n. ${ }^{\circ} 11.070(08 / 11 / 1915)$, n. ${ }^{\circ} 13.563(07 / 06 / 1919)$, n. $^{\circ} 13.997$ (21/11/1919), n. ${ }^{\circ} 14.040(09 / 12 / 1919),{ }^{\circ}{ }^{\circ} 15.413(01 / 09 / 1921)$, n. ${ }^{\circ} 16.458(08 / 01 / 1923)$, n. $^{\circ} 16.052(07 / 08 / 1922)$, n. $^{\circ} 16.259(25 / 10 / 1922)$.

88 Ingreso n. ${ }^{\circ} 10.277(14 / 09 / 1914)$.

89 Ingreso n. ${ }^{\circ} 10.078(13 / 06 / 1914)$. 
El cambio histórico de estas prácticas sobre la niñez fue un proceso que tardó décadas. En ese marco, solo algunos ingresos indicaban cuidados en ciertos niños. Por ejemplo, Rosa de Jesús «vuelve a la sección Lactancia por ser muy raquítica, hasta que se desarrolle más». ${ }^{90} \mathrm{La}$ información apuntada en los libros sobre los recién nacidos recopilaba las malas condiciones de salud en el momento de la entrada. En ellos se explicitó que un niño ingresó «agonizante de hambre» ${ }^{91} \mathrm{o}$ una niña de 5 años fue «traída de Temuco. Llegó quebrada por raquitismo». ${ }^{92}$ Otras referencias sobre la falta de alimentación en los niños fueron introducidas en documentos judiciales y periodísticos.

El brote de viruela de 1904 evidenció la implementación de tratamientos médicos preventivos en la salud infantil, vacunándose «por primera vez, 180 niños». ${ }^{93}$ De igual manera, la Junta de Beneficencia podía modificar los tratamientos médicos que prestaba la Casa de Huérfanos durante los brotes epidémicos de influenza. En 1918 fueron ocupadas 906 camas del establecimiento y 253 de la sección talleres. Ambas fueron parte de las 4.472 que se dispusieron para recibir a los enfermos. ${ }^{94}$

Como señalamos al inicio de esta investigación, en 1928 se efectuaron una serie de reformas que mejoraron la condición en la que se encontraban los niños en el interior de la Casa. En el aspecto médico, se reorganizaron los servicios, «se creó el internado, se reorganizó la botica, se creó un curso obligatorio para las enfermeras y ayudantes, se reorganizó la vigilancia nocturna del servicio de lactantes; se higienizó el establo y se organizó la cocina de leche; se han establecido laboratorios biológicos y de Rayos X». ${ }^{95}$ Se intentaba limitar el número de internados y facilitar la entrega de los infantes a las nodrizas para su alimentación. Esta disminución de lactantes internados en la Casa de Huérfanos permitió habilitar nuevos lavatorios y baños que habían desaparecido para albergar mayor cantidad de camas. Además, el propósito de estas transformaciones era conformar un sistema de inspección médico y social que efectuara la difusión de los principios de la puericultura entre las familias que acogían infantes.

90 Ingreso n. ${ }^{\text {o }} 14.041(09 / 12 / 1919)$.

91 Ingreso n. ${ }^{\circ} 16.263(25 / 10 / 1922)$.

92 Ingreso n. ${ }^{\circ} 12.671(08 / 03 / 1918)$.

93 «La viruela en Santiago», Santiago, El Mercurio, 11 de febrero de 1904, 5.

94 «La epidemia de influenza permanece estacionaria», El Mercurio, Santiago, 21 de noviembre de 1918,3 .

95 «Reforma integral de la Casa de Huérfanos. La dirección del establecimiento explica a la Junta su programa», El Mercurio, Santiago, 13 de noviembre de 1928, 12. 
Para el caso de la Casa de Huérfanos, la incorporación de médicos especialistas significó la disminución de la mortalidad infantil por varias razones, entre ellas: la separación entre niños enfermos y sanos, las observaciones científicas que permitían tratar las enfermedades, la difusión de los principios de lo que denominaron maternidad científica y mejoras en las prácticas de alimentación. A ello se agregó la función que cumplió la Junta de Beneficencia mediante la instalación de Gotas de Leche, Asilos Maternales, el Patronato de la Infancia y otros organismos que contribuyeron en la nutrición y la difusión de las nociones de cuidados en los recién nacidos.

\section{Conclusiones}

La historia de la Casa de Huérfanos durante 1898 y 1923 verificó la hipótesis de que el fenómeno de la mortalidad infantil en la citada institución se enmarcó dentro de los profundos cambios científicos (progresos de la medicina infantil) y culturales (narrativas nuevas del abandono). El análisis de los datos demostró cómo el desarrollo de un conocimiento médico dedicado a los niños se tradujo en la disminución de la mortalidad de ese grupo. Lo anterior, cabe señalar, no se logró sin un abordaje científico y sanitario que estableciera planes para disminuir las principales causas de las muertes (alimentación y cuidados infantiles deficientes).

Para abordar el problema de la mortalidad infantil en la Casa de Huérfanos, describimos y analizamos cuatro aspectos donde se desarrolló esa experiencia. En primer lugar, el abandono de párvulos sanos o enfermos en diversos lugares de Santiago, lo que produjo importantes debates médico-legales que mejoraron, en la práctica, la sanidad del establecimiento, mientras que paralelamente los diagnósticos de los internados evolucionaban desde una mirada religiosa hacia otra «científica». En segundo lugar, los modelos de crianza ofrecidos por las familias contratadas por la Casa, considerando que algunas de aquellas experimentaron la muerte de los menores a raíz de accidentes o malos tratos. En tercer lugar, los descuidos maternales como causa precisa de muertes. Asimismo, varias familias entregaron niños a la Casa, los que posiblemente fallecieron ahí. Por último, y no menos importante, los trabajos de médicos y políticos evidencian el fracaso relativo de esa institución en el control de la mortalidad de niños hasta, por lo menos, el advenimiento de la pediatría chilena. 
Metodológicamente, los registros de la Casa de Huérfanos permitieron la construcción de una estadística detallada de los ingresos anuales, los que conforman una muestra de los preceptos discutidos por la medicina y de los valores culturales de la sociedad. Estas dos ideas matrices explicaron la elevadísima mortalidad en la institución. En el caso de la primera, relacionándola con la condición de raquitismo de los niños, la edad temprana y el aumento de muertes durante el verano. Para la segunda, podemos aseverar que la mirada conservadora relacionó el abandono con la ilegitimidad y la ruptura familiar. De ese modo, constatamos una preocupación moral sobre una práctica médica, la que explica, en parte, la mortalidad infantil dentro de la Casa. Eso pudo inducir a pensar que los recién nacidos fallecieron por tales causas. Pero, en concreto, los infantes murieron por la escasez de higiene y tratamientos médicos de las enfermedades, así como por la ausencia de medidas profilácticas (por ejemplo, separando niños sanos de los enfermos para evitar contagios).

En este proceso de medicalización distinguimos las características de la mortalidad infantil en un régimen de internación. En ese contexto, el establecimiento a cargo de la Junta de Beneficencia permite visualizar varios discursos sobre este problema. Entre los actores involucrados pudimos distinguir a las religiosas encargadas de llevar los registros, quienes desarrollaron una mirada moral; los médicos, que propusieron mejoras en los cuidados de los recién nacidos, y, finalmente, quienes testimoniaron al momento de la internación de los menores. A partir de estos testimonios trazamos el surgimiento de una medicina dedicada a la infancia en la Casa de Huérfanos que se difundió entre facultativos, nodrizas y mujeres. Ese saber transcurrió entre las tensiones propias del encuentro entre la esfera científica, por un lado, y popular, por otra.

Esta investigación aporta nuevas propuestas de trabajo que enriquecen la historiografía chilena de la infancia. El análisis de estos registros permite captar las ideas existentes en dicho contexto sobre la niñez, lo circunstancial del abandono y las justificaciones de los testigos. Sobre este aspecto se puede inferir un predominio de decisiones de las madres, quienes, en la mayoría de los casos, efectuaban el traslado de los niños hacia la institución, acusaban el abandono de sus maridos e, incluso, el rechazo familiar de sus maternidades.

Este campo en formación del conocimiento médico proyectó sus saberes y preceptos en la población santiaguina. La generación de este modelo se apreció en los avances de los tratamientos y el desarrollo de los cuidados 
infantiles que estuvieron sustentados en una disminución de la mortalidad. Este período resignificó la niñez, iniciándose transformaciones que, a mediados del siglo XX, devinieron en la reducción de la desnutrición, la medicalización de la infancia y el aumento de la esperanza de vida.

Recibido el 3 de agosto de 2017 Segunda versión 7 de marzo de 2018 Tercera versión 12 de junio de 2018 Aceptado el 18 de julio de 2018

\section{Referencias bibliográficas}

Aliaga, Fernando, Historia de la Congregación Hermanas de la Providencia en Chile, t. IV., Santiago, Sociedad Impresora La Unión Ltda., 1993.

Álvarez, Adriana y Reynoso, Daniel, «Entre el abandono y la debilidad. El cuidado de la salud en la primera infancia, 1920-1930», en Cosse, Isabella et al., Infancias: políticas y saberes en Argentina y Brasil. Siglos XIX y XX, Buenos Aires, Editorial Teseo, 2011, 123-149.

Arévalo, Francisco, El abandono en la infancia, Memoria de prueba para optar al grado de Licenciado en la Facultad de Leyes y Ciencias Políticas, Santiago, Universidad de Chile, 1918.

Blum, Ann, Domestic Economies: Family, work and welfare in México city, 1884 1943, Lincoln, University of Nebraska, 2010.

Boswell, John, The Kindness of Strangers: the abandonment of children in Western Europe from late antiquity to Renaissance, New York, Pantheon Books, 1988.

Calvo Mackenna, Luis, «Lo que deben saber las madres para criar bien a sus niños», Primer Congreso Nacional de Protección a la Infancia. Trabajos y actas, Santiago, Imprenta Barcelona, 1913, 5-20.

Calvo Mackenna, Luis, Memoria de la Casa Nacional del Niño. Breve reseña de su labor desde 1927 a 1933, inclusive, Santiago, Imprenta Casa Nacional del Niño, 1933.

Cordero Baños, Eduardo, Los males sociales como causa de la mortalidad infantil, Memoria de prueba para optar al grado de Licenciado en Medicina, Santiago, Imprenta Fiscal de la Penitenciaría, 1923.

Correa, María José, «Cuerpo y demencia. La fisonomía de la incapacidad en Santiago de Chile (1855-1900)», Historia Crítica, 46, Bogotá, 2012, 88-109.

Correa, María José, «De la Casa de Orates al Juzgado. Pericia alienista y evaluación judicial de la locura en Santiago de Chile hacia 1860», História, Ciências, Saúde-Manguinhos, 20, 2, Río de Janeiro, 2013, 571-585.

Cowen, Miguel Pablo, «Infancia, abandono y padres en el s. XIX porteño», Аnиаrio del Instituto de Historia Argentina, 4, Buenos Aires, 2004, 75-99. 
Dalla-Corte, Gabriela y Piacenza, Paola, A las puertas del hogar. Madres, niños y damas de Caridad en el Hogar del Huérfano de Rosario (1870-1920), Rosario, Prohistoria Ediciones, 2006.

Dalla Corte, Gabriela, El archivo de señales del Hogar del Huérfano de Rosario: niñez, identidad y migración 1879-1914, Rosario, Prohistoria Ediciones, 2013.

Del Castillo Troncoso, Alberto, Conceptos, imágenes y representaciones de la niñez en la Ciudad de México 1880-1920, Ciudad de México, El Colegio de México, Centro de Estudios Históricos, Instituto de Investigaciones Dr. José María Luis Mora, 2009.

Del Río, Roberto y Commentz, Alfredo, Anotaciones sobre la protección a la infancia en Chile. Condiciones de producción de leche destinada a la alimentación de niños. Relación presentada al primer Congreso Científico Panamericano, Santiago, Imprenta Universitaria, 1909.

Delgado Valderrama, Manuel, «La infancia abandonada en Chile, 1770-1930», Revista de historia social y de las mentalidades, 5, Santiago, 2001, 101-126.

Díaz, José, Gallego, Francisco y Lafortune, Jeanne, «Nacimientos fuera del matrimonio en la historia de Chile: algunos hechos estilizados», Estudios públicos, 142, Santiago, 2016, 37-79.

Foucault, Michel, La vida de los hombres infames, Buenos Aires, Editorial Altamira, 1996.

Franco, Renato, «Discriminação e abandono de recém-nascidos mestiços na América Portuguesa: Os exemplos de Mariana, Vila Rica e Recife», Varia Historia, 32, 59, Belo Horizonte, 2016, 437-469.

González, Fabio, «Niñez y beneficencia. Un acercamiento a los discursos y estrategias disciplinarias en torno a los niños abandonados en Buenos Aires de principios del siglo XX (1900-1930)», en Moreno, José Luis, La política social antes de la política social. Caridad, beneficencia y política social en Buenos Aires, siglos XVII a XX, Buenos Aires, Prometeo, 2000, 129-204.

Guy, Donna, White slavery and mothers alive and dead: the troubled meeting of sex, gender, public health and progress in Latin America, Lincoln, University of Nebraska, 2000.

Herrera Rogers, Jorge, «Protección médica y social del niño de pecho indigente», Primer Congreso Nacional de Protección a la Infancia. Trabajos y actas, Santiago, Imprenta Barcelona, 1913, 53-68.

Hunecke, Volker, «Intensità e fluttuazioni degli abbandoni dal XV al XIX secolo», Enfance abandonée et société en Europe, París, Publications de l'École Française de Rome, 1991, 27-72.

Ipinza Besoaín, Arnaldo, De la natalidad ilegítima. Sus causas y modo de remediarlas, Memoria de prueba para optar al grado de Licenciado en la Facultad de Leyes y Ciencias Políticas, Santiago, Universidad de Chile, 1920. 
Korner, Víctor, «Protección de las madres durante el embarazo, el parto y el puerperio, y su influencia sobre la mortalidad infantil», Primer Congreso Nacional de Protección a la Infancia. Trabajos y actas, Santiago, Imprenta Barcelona, 1913, 135-144.

Letelier, Hipólito, La protección de la infancia, Memoria de prueba para optar al grado de Licenciado en Leyes y Ciencias Políticas, Santiago, Universidad de Chile, 1918.

Lisboa Huerta, Jenaro, Mortalidad de párvulos en Chile: sus causas, modo de evitarlas, Memoria de prueba para el examen de Licenciado en la Facultad de Medicina, Santiago, Universidad de Chile, 1886.

Martín-Espinosa, Noelia, Villena Espinosa, Rafael y Cobo-Cuenca, Ana, «Análisis de la mortalidad expósita de la Inclusa de Toledo y sus condicionantes (19001930)», Asclepio. Revista de Historia de la Medicina y de la Ciencia, 68, 1, Madrid, 2016, 130-145.

Matte, Eugenio, Natalidad ilegítima en Chile, Santiago, Imprenta y Encuadernación «Claret», 1918.

Milanich, Nara, «Los hijos de la Providencia: el abandono como circulación en el Chile decimonónico», Revista de historia social y de las mentalidades, 5, Santiago, 2001, 79-100.

Milanich, Nara, "“Casa de Huérfanos" and child circulation in late-nineteenth century Chile», Journal of Social History, 38, 2, Oxford, 2004, 311-340.

Milanich, Nara, Children of fate. Childhood, class, and the State in Chile, 18501930, Durham, Duke University Press, 2009.

Milanich, Nara, «Women, children, and domestic labor in nineteenth-century Chile», Hispanic American Historical Review, 91, 1, Durham, 2011, $29-62$.

Morín Rouleau, Bernarda, Historia de la Congregación de las Hermanas de la Providencia de Chile, Santiago, Imprenta de San José, 1899.

Olivares, Luis, La mortalidad infantil y sus causas en la Casa de Expósitos de Santiago, Memoria de prueba para optar al grado de Licenciado en la Facultad de Medicina y Farmacia, Santiago, Universidad de Chile, 1892.

Osta Vásquez, María Laura, «Niños y niñas, expósitos y huérfanos en Montevideo del siglo XIX», Revista de la Facultad de Derecho, 41, Buenos Aires, 2016, 155-189.

Palma, Patricia, «Mortalidad infantil en Chile. La situación médica social en la Casa de Expósitos de Santiago 1889-1899», Anales de historia de la medicina, 19, 2, Santiago, 2009, 127-137.

Ponce de León, Macarena, La reforma de la caridad ilustrada: del socorro intramuros al socorro extramuros. Prácticas de caridad en Santiago, 1830-1880, Tesis para optar al grado de Doctor en Historia, Santiago, Pontificia Universidad Católica de Chile, 2007.

Pulido Illanes, David, «Vulgarización de la puericultura y de la alimentación de los niños en el público», Primer Congreso Nacional de Protección a la Infancia. Trabajos y actas, Santiago, Imprenta Barcelona, 1913, 101-112. 
Quay Hutchinson, Elizabeth, «La historia detrás de las cifras: la evolución del censo chileno y la representación del trabajo femenino, 1895-1930», Revista Historia, 33, Santiago, 2000, 417-434.

Revuelta Eugercios, Bárbara, Los usos de la inclusa de Madrid, mortalidad y retorno a principios del siglo XX (1890-1935), Madrid, Tesis doctoral Universidad Complutense de Madrid, Facultad de Geografía e Historia, 2011.

Revuelta, Bárbara, «Releasing mother's burdens: Child Abandonment and Retrieval in the Foundling Hospital of Madrid, 1890-1935», Journal of Interdisciplinary History, XLII, 4, Cambridge, 2012, 645-672.

Revuelta, Bárbara, «Abandoned and illegitimate, a double mortality penalty? Mortality of illegitimate infants in the foundling hospital of Madrid, La Inclusa (1890-1935)», Journal The History of the Family, 18, California, 2013, 44-67.

Revuelta Eugercios, Bárbara, «¿Qué pasa en La Inclusa? The role of press scandals, doctors and public authorities in the evolution of La Inclusa de Madrid, 1890-1935», Dynamis, 35, 1, Barcelona, 2015, 107-130.

Rodríguez, Esteban, «La construcción de la salud infantil. Ciencia, medicina y educación en la transición sanitaria en España», Cuadernos de Historia Contemporánea, 18, Madrid, 1998, 19-52.

Rodríguez, Pablo y Manarelli, María Emma, Historia de la infancia en América Latina, Colombia, Universidad Externado de Colombia, 2007.

Román Añibar, Julio, Mortalidad de niños menores de un año en Santiago de Chile. Estadística, causas i profilaxia 1895-1899, Memoria de prueba para optar al grado de Licenciado en la Facultad de Medicina y Farmacia, Santiago, Imprenta y encuadernación «El Globo», 1900.

Salinas, René y Delgado, Manuel, «Los hijos del vicio y del pecado: la mortalidad de los niños abandonados (1750-1930)», Proposiciones, 19, Santiago, 1990, 44-54.

Salinas, René, «Orphans and family disintegration in Chile: the mortality of abandoned children, 1750-1930», Journal of family history, 16, 3, California, 1991, 315-329.

Sánchez Calleja, María Eugenia, Niños y adolescentes en abandono moral. Ciudad de México (1864-1926), México, Instituto Nacional de Antropología e Historia, 2014.

Sánchez Cruz, Alfredo, «La alimentación en la primera infancia», Primer Congreso Nacional de Protección a la Infancia. Trabajos y actas, Santiago, Imprenta Barcelona, 1913, 69-92.

Simon, Robert, «Reducción de la mortalidad infantil del primer año por la asistencia a las madres antes, durante y después del parto», Primer Congreso Nacional de Protección a la Infancia. Trabajos y actas, Santiago, Imprenta Barcelona, 1913, 219-224. 
Vargas Guerra, Eduardo, La ilegitimidad en Chile i sus consecuencias, Memoria de prueba para optar al grado de Licenciado en la Facultad de Leyes y Ciencias Políticas, Santiago, Imprenta Camilo Henríquez, 1917.

Villarroel, Julio, Beneficencia, Memoria para optar al grado de Licenciado en la Facultad de Leyes y Ciencias Políticas, Santiago, Imprenta Aurora, 1906.

Zárate, María Soledad, Dar a luz en Chile, siglo XIX, Santiago, Ediciones Universidad Alberto Hurtado, 2007. 


\section{PABLO CHÁVEZ ZÚÑ̃IGA Y JOSÉ JULIÁN SOTO LARA}

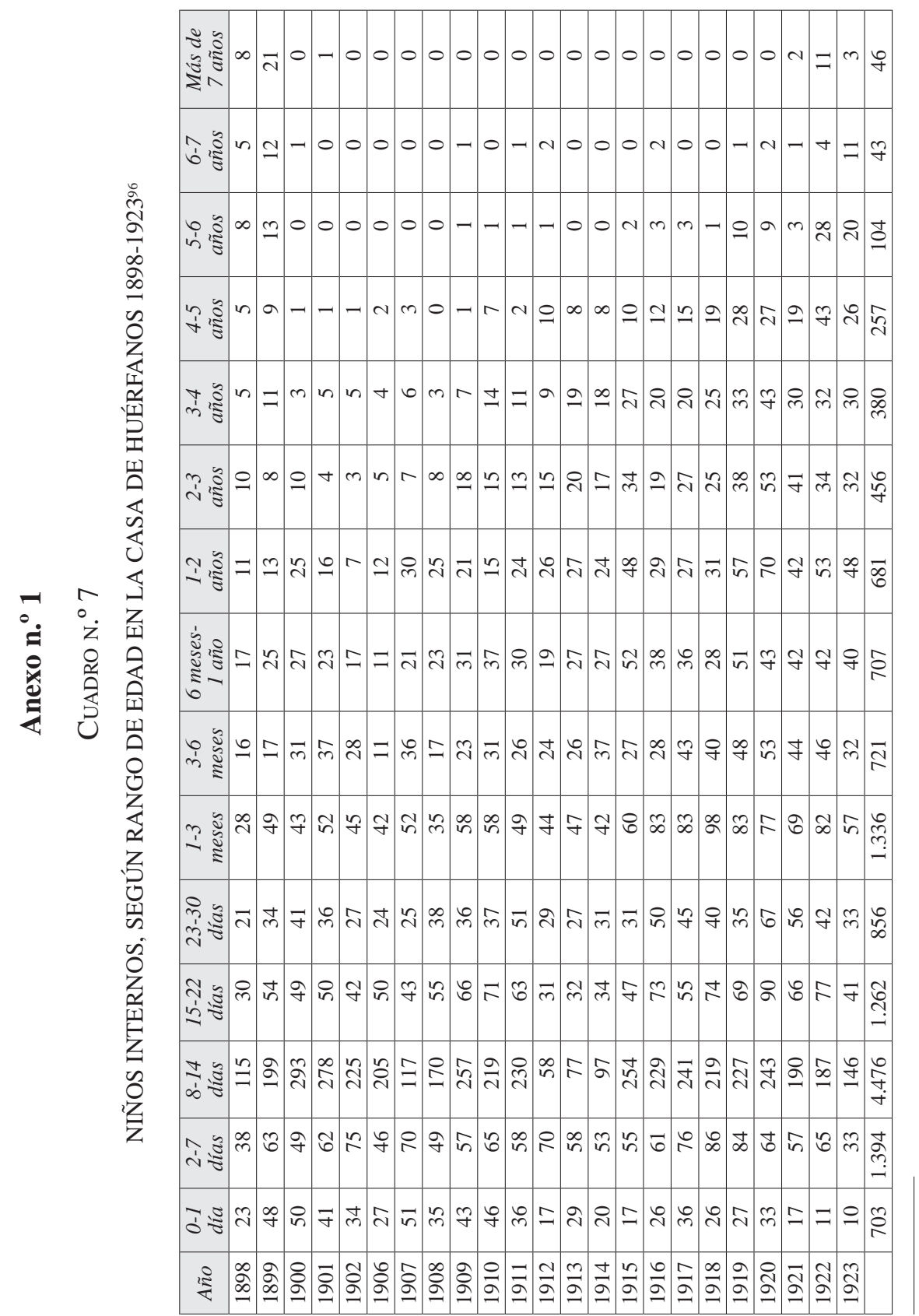




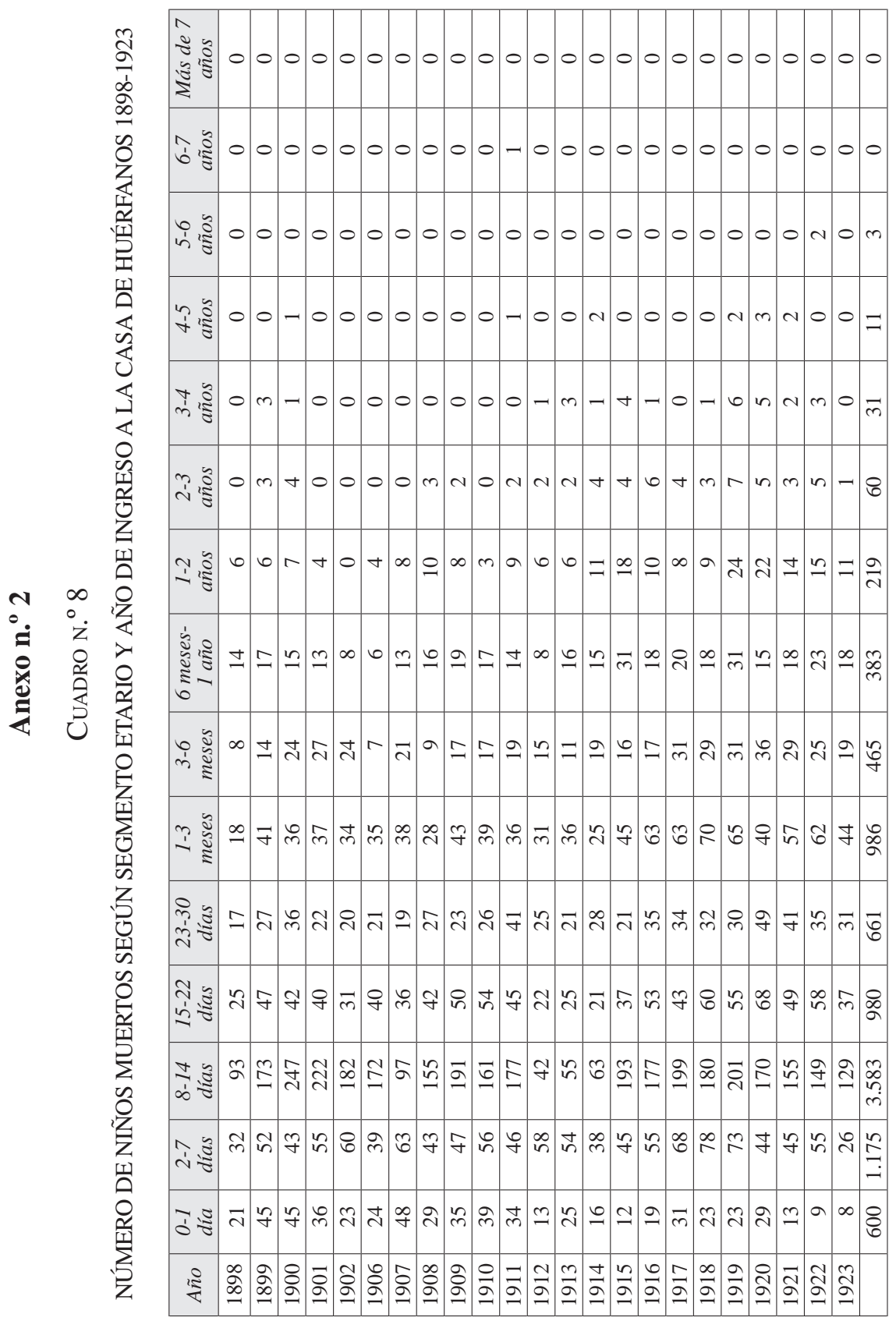

